Cahiers de recherches médiévales

Journal of medieval studies

5 | 1998

Le choix de la prose (XIIIe-XVe siècles)

\title{
Des Sarrasins à Camaalot
}

\section{Carol Chase}

\section{(2) OpenEdition}

Journals

Édition électronique

URL : https://journals.openedition.org/crm/1372

DOI : $10.4000 / \mathrm{crm} .1372$

ISSN : 1955-2424

\section{Éditeur}

Honoré Champion

\section{Édition imprimée}

Date de publication : 30 octobre 1998

Pagination : 43-53

ISSN : 1272-9752

\section{Référence électronique}

Carol Chase, "Des Sarrasins à Camaalot», Cahiers de recherches médiévales [En ligne], 5 | 1998, mis en ligne le 01 octobre 2007, consulté le 15 décembre 2022. URL : http://journals.openedition.org/crm/ 1372 ; DOI : https://doi.org/10.4000/crm.1372

Ce document a été généré automatiquement le 15 décembre 2022.

Tous droits réservés 


\section{Des Sarrasins à Camaalot}

\section{Carol Chase}

1 Dans un des récits rétrospectifs qui ramènent le lecteur du Lancelot en prose à la première ère chrétienne, le narrateur décrit la Grande Bretagne comme habitée par des Sarrasins : «ne il n'avoit a cel termine en la Grant Bretaigne se Sarrazins non. » Même Camaalot, haut lieu de la géographie arthurienne, est occupée par les infidèles, selon l'histoire de la Croix Noire, croix qui est restée noire du sang de douze compagnons de Joseph d'Arimathie martyrisés par le roi cruel de cette ville, Agrestes (II, 321). Ce passage fait partie d'une série de références et de «flashbacks» qui s'inscrivent au temps où « li gentils chevaliers » Joseph d'Arimathie a combattu les Sarrasins en Grande Bretagne. ${ }^{2}$

2 Cette étonnante vision d'une Grande Bretagne sarrasine est reprise et élaborée par l'Estoire del Saint Graal, l'ouvrage qui sert de porte d'entrée au cycle du Lancelot-Graal. Lorsque Joseph d'Arimathie et les chrétiens qui l'ont suivi depuis Jérusalem arrivent en Grande Bretagne, ils « virent la terre et le paiis qui toz estoit pueplez de sarrazins et de mescreanz. $»^{3} \mathrm{Ce}$ roman, écrit après le Lancelot, peut être vu comme une vaste histoire rétrospective par rapport aux autres parties du cycle :4 il raconte les origines du Graal et sa "translatio » de Jérusalem en Grande Bretagne par Joseph d'Arimathie aussi bien que les origines des lignages des grands héros arthuriens. Dans ce retour aux racines l'Estoire utilise la technique de la réécriture - technique commune à tout le cycle - mais à tel point que l'on pourrait comparer le roman à un «collage » ou un patchwork. Nous utilisons cette métaphore pour insister sur la cohérence générale du texte, car comme le travail des artistes cubistes ou des créateurs de patchwork, c'est un ensemble formé de morceaux soigneusement choisis et collés ou cousus pour créer un dessin dans lequel chaque forme prend un nouveau sens. ${ }^{5}$ Ainsi l'Estoire reprend-elle le récit de la Croix Noire presque textuellement et l'insère dans le cadre général du voyage évangélisateur de Joseph d'Arimathie, de son fils Josephé et du groupe de chrétiens qui partent de Jérusalem pour se rendre dans la Nouvelle Terre Promise, l'Angleterre. Ce nouveau milieu donne un nouveau sens à cette histoire.

3 Dans le Lancelot le récit de la Croix Noire est pris en charge par le narrateur, qui interrompt l'histoire d'une quête de Lancelot par Gauvain et ses compagnons pour 
expliquer la présence de cet objet dans le paysage arthurien. Peu après cette pause narrative les quêteurs rencontrent un chevalier qui porte deux épées dont l'une est brisée. Celui-ci propose à Gauvain et ses compagnons d'essayer de ressouder l'épée, puis il leur en raconte l'histoire. ${ }^{6}$ Cette histoire est également reprise par l'Estoire. ${ }^{7}$ Dans le Lancelot Joseph d'Arimathie est au centre de ces deux récits, qui démontrent le triomphe du christianisme sur la foi sarrasine. La croix et l'épée servent donc de point de départ pour des narrations qui évoquent le passé pré-arthurien et qui expliquent le sens de ces deux objets significatifs par l'histoire de leur origine.

Dans l'Estoire en revanche les deux récits sont insérés dans l'intrigue de façon chronologique - ils font partie de toute une série d'épisodes concernant l'évangélisation de la Grande Bretagne. Les objets annoncent l'avenir dans la grande fresque historique du temps arthurien qu'est le cycle du Lancelot-Graal. Le narrateur affirme que la croix noire restera noire du sang des douze martyrs, tandis que l'épée, qui s'est brisée lorsqu'un Sarrasin en a frappé Joseph à la cuisse, ne sera ressoudée que par celui qui doit terminer «les hautes aventures del Graal» (II, 501). En plus, dans la première de ces deux histoires, l'auteur anonyme de l'Estoire substitue à Joseph d'Arimathie un nouveau personnage, le fils de ce dernier, Josephé. L'invention de ce personnage est liée à la réécriture et à l'amplification de l'histoire de Joseph d'Armathie telle qu'elle parait dans Le Roman de l'Estoire del Graal de Robert de Boron aussi bien que dans ces deux récits. ${ }^{8}$ Dans le Lancelot Joseph est présenté comme le premier chevalier chrétien; le rôle qu'il joue est celui d'un guerrier aussi bien que celui d'un apôtre. ${ }^{9}$ Mais dans les récits de la Croix Noire et de l'épée brisée, le narrateur insiste sur son rôle d'apôtre et c'est cet aspect qui sera élaboré par l'Estoire. ${ }^{10}$ Afin d'amplifier les aventures de Joseph, le prosateur utilise deux procédés. D'une part, le personnage de Joseph est scindé en deux. ${ }^{11}$ Joseph partage avec son fils Josephé le rôle évangélisateur; il est même éclipsé par son fils qui devient le guide spirituel des chrétiens. D’autre part, l'insistance sur cette fonction entraîne la multiplication d'épisodes similaires à ceux qui sont développés dans les histoires de la Croix Noire et de l'épée brisée, où des Sarrasins sont convertis au christianisme.

Notre but ici n'est pas d'étudier tout le travail de réécriture de l'auteur de l'Estoire. Ce qui va retenir notre attention, c'est l'amplification du rôle des Sarrasins. Des Sarrasins en Grande Bretagne et même à Camaalot? Des Sarrasins dans un roman en prose arthurien? Le lecteur pourrait croire qu'il s'est perdu et qu'il se promène dans une chanson de geste, en Espagne ou en Orient. Cette vision étonnante mérite un détour de la part du critique. Nous nous proposons donc d'étudier les Sarrasins dans l'Estoire del Saint Graal, en faisant des comparaisons avec leur peinture dans la chanson de geste, en particulier une chanson du cycle de Guillaume d'Orange qui est contemporaine de notre roman en prose, c'est-à-dire du début du XIII ${ }^{e}$ siècle, les Enfances Guillaume. ${ }^{12}$ Nous avons choisi ce texte parce que, comme l'Estoire, il représente un retour en arrière narratif par rapport au reste du cycle, parce que la présentation des Sarrasins y est particulièrement intéressante et parce que c'est une chanson qui a été peu étudiée. Nous nous tournerons aussi vers la Chanson de Roland, bien qu'elle soit antérieure à notre texte, parce qu'elle présente un large éventail de tous les thèmes de la chanson de geste en ce qui concerne les Sarrasins et qu'elle est le point de repère par excellence.

6 L'Estoire del Saint Graal est structurée par le voyage de Joseph d'Arimathie, de son fils Josephé et des premiers chrétiens d'Orient en Occident. Pendant ce périple Joseph et Josephé prêchent, font des débats théologiques, guérissent, convertissent et baptisent 
toute une série de souverains païens ou sarrasins. Il faut noter que le prosateur utilise les deux termes païen et sarrasin de façon interchangeable pour désigner les ennemis des chrétiens - dont la religion est assimilée à l'Islam - et qu'il confond des pratiques païennes avec celles de la religion musulmane, comme dans la chanson de geste. ${ }^{13}$ Nous verrons que cette confusion a des raisons idéologiques.

7 Le premier volet de l'Estoire est consacré à la conversion du roi Évalach et de sa famille. Rappelons qu'au moment du baptême Évalach prend le nom de Mordrain et son beaufrère, Seraphe, celui de Nascien. L'action est située à Sarras et dans les environs de cette ville orientale. Dans la partie centrale du roman Évalach-Mordrain, SerapheNascien et le fils de ce dernier, Celidoine, sont transportés dans des îles ou dans des bateaux où leur foi est mise à l'épreuve. La narration passe sous silence les aventures des chrétiens après leur départ de Sarras jusqu'au moment où ils traversent la mer pour arriver en Grande Bretagne. Dans la dernière partie du roman tous les personnages sont réunis en Grande Bretagne, où Joseph et Josephé continuent leur activité évangélisatrice, convertissant toute une série de souverains et de nobles sarrasins et leur peuple.

Chacune des conversions est caractérisée par un certain nombre de traits et d'étapes qui se répètent avec des variations. La conversion d'Évalach, qui est la plus compliquée et qui occupe une grande partie du roman, nous servira de modèle. On peut y déceler les étapes suivantes : 1 . leçon de Joseph sur l'histoire chrétienne ; 2. prière de Joseph ; 3. visions du roi ; 4. débat de Josephé avec un «clerc» sarrasin - interrompu par la punition de celui-ci, qui a parlé contre la Trinité ; 5 . démonstration de l'inefficacité des dieux sarrasins et destruction des idoles; 6 . révélations par Josephé sur les origines et l'enfance d'Évalach; 7.guerre contre les Égyptiens (victoire d'Évalach après l'intervention du Christ); 8. guérison d'un chevalier dont la main a été coupée (Clamachidés) ; 9. conversion et baptême de Seraphe-Nascien puis d'Évalach-Mordrain et des habitants de leurs terres; 10. châtiment de ceux qui refusent d'adopter le christianisme; 11. destruction des temples sarrasins et fondation des églises chrétiennes; 12. révélation des mystères du Graal; 13. explication des visions d'Évalach ; 14. destruction d'une poupée-idole qu'Évalach gardait en secret.

Cette conversion, qui a lieu à Sarras, ville orientale que le narrateur identifie avec les origines des Sarrasins, comporte un grand nombre de séquences narratives. Les épisodes de conversion qui ont lieu en Grande Bretagne reprennent ce modèle, en le simplifiant, omettant certaines étapes et variant leur ordre. Les commentaires qui suivent s'appliquent donc à toutes les histoires de conversion.

10 Il est notable que tous les moyens sont bons pour convaincre Évalach d'adopter la foi chrétienne. En fait, sa conversion semble acquise par avance : Dieu a "pré-sélectionné » certains souverains pour faire partie du nouveau peuple qui va fonder les lignages des héros arthuriens. S'il y a souvent des guerres qui accompagnent ou qui suivent les conversions, ni Joseph ni son fils Josephé ne jouent un rôle militaire dans ces actions. Ce sont les nouveaux convertis qui agissent comme des guerriers chrétiens. La campagne qu'Évalach mène contre les Égyptiens n'a pas pour but de convertir ces derniers mais plutôt d'éprouver le roi, qui résiste à la conversion. Dans les expéditions militaires en Grande Bretagne, le but est d'anéantir les Sarrasins qui sont rebelles au christianisme. Il s'agit bien de guerres saintes, comme dans la chanson de geste, car lors d'une bataille contre le roi de Northumberland, qui assiège son vassal, le duc Ganor, 
nouvellement converti, Seraphe-Nascien assure ce dernier que s'ils meurent, «ce nos sera sauvemenz, puis que nos por la loi Jesucrist recevrons martire » (II, 449).

Quant à Joseph et Josephé, leur lutte est plutôt verbale : ils utilisent la persuasion pour obtenir des conversions. Cependant, la violence n'est pas absente de ces épisodes : ceux qui refusent le christianisme sont systématiquement punis. Les clercs sarrasins qui s'élèvent contre les doctrines chrétiennes sont châtiés de façon exemplaire: ils commencent à mugir et perdent la capacité de parler. Les autres récalcitrants sont punis de diverses façons. Par exemple, le duc Ganor, nouvellement converti, chasse de sa ville les habitants qui n'ont pas voulu embrasser le christianisme. Ceux-ci montent dans un bateau; pendant la nuit il y a une grande tempête et tous meurent noyés. Le but des chrétiens n'est pas une coexistence des deux religions mais l'anéantissement de la religion sarrasine ${ }^{14}$ La tolérance est inexistant - attitude que l'on trouve aussi dans la chanson de geste.

Il faut noter que les nouveaux chrétiens dont la foi n'est pas assez ferme sont aussi l'objet de châtiments exemplaires. Dans toute une série d'épisodes qui alternent avec ceux où la conversion des Sarrasins est obtenue, les chrétiens qui doutent ou qui transgressent des lois sont punis. On peut citer à titre d'exemple la punition de Moïse lorsqu'il s'assoit à la place vide à la table du Graal, malgré l'avertissement de Josephé qu'elle représente l'endroit où s'asseyait le Christ à la Cène : sept mains en flammes descendent du ciel et l'emportent (II, 487).

Le combat des chrétiens contre les Sarrasins est idéologique. Michelle Szkilnik a montré comment deux articles de la foi chrétienne marquent le tissu narratif de l'Estoire: la Trinité et la virginité de Marie.$^{15}$ Le rôle important de la Trinité apparait dès la première phrase de l'Estoire: le narrateur ouvre le prologue en s'adressant à «tous cheus et a toutes cheles ki ont lor creanche en la sainte, glorieuse Trinité » (I, 1) ${ }^{16}$ Dans la première partie du prologue il raconte comment Dieu l'a rassuré sur la Trinité, le seul article de foi qui lui ait causé de doutes.

14 C'est aussi la Trinité qui soulève des doutes chez les Sarrasins dans l'Estoire. Dans les discussions théologiques qui précèdent certaines conversions, les Sarrasins expriment leur scepticisme en ce qui concerne ce dogme aussi bien que sur la virginité de Marie, et il faudra les convaincre sur ces points. Lorsque Joseph expose ces doctrines à Évalach, ce dernier répond, «Tu me fais entendant unes coses ke nus ne porroit metre en voir ne en nule maniere ne samble raisons, car tu dis ke il (Jésus) ne fu pas engenrés en la feme dont il nascui, et ke ele estoit puchiele ne onques ses puchelages n'en empira : aprés me dis ke li Peres et li Fiex et li Sains Esperis ne sont ke uns seus Diex, et si est chascuns d'aus .III. Diex par soi» (I, 57). Évalach utilise ici un langage logique et rationnel; la réponse de Joseph sera fondée plutôt sur le «langage du cœur» ou de la foi : «Tu l'as bien recordé ainsi com je le t'ai dit, et ensi le tesmoigne jou bien encore » $(\mathrm{I}, 57) \cdot{ }^{17}$

15 En fait, tous les débats théologiques sont caractérisés par cette opposition. D'ailleurs ce sont des débats boiteux, car les Sarrasins parlent peu. Ils sont silencieux sur leurs propres doctrines religieuses et se contentent d'écouter les leçons des chrétiens en posant parfois des questions ou en exprimant leurs doutes. Dans ces «discussions» religieuses, afin de convaincre les Sarrasins, les chrétiens racontent l'histoire biblique et expliquent la doctrine. Les discussions sont complétées par des guérisons miraculeuses et par des visions envoyées par Dieu et interprétées par Joseph ou 
Josephé. Les visions et leur interprétation recourent à l'analogie afin de faire comprendre les mystères chrétiens. Quant aux vrais débats théologiques avec les «clercs» ou philosophes sarrasins, ils sont toujours coupés net: Dieu intervient pour faire taire le clerc qui ose parler contre le dogme chrétien. ${ }^{18}$

L'Estoire accorde beaucoup d'importance aussi à la croix, signe du christianisme. Cet objet hautement symbolique est évoqué à maintes reprises tout le long du roman. À titre d'exemple on peut citer la Croix Noire que nous avons déjà mentionnée et sur laquelle nous reviendrons plus loin et l'écu à la croix vermeille que Josephé prépare pour Évalach lorsqu'il part à la guerre contre les Égyptiens. Cet écu, qui va jouer un rôle important dans l'Estoire aussi bien que dans la Queste, montre la valeur symbolique de la croix..$^{19}$

17 L'importance de la Trinité et de la croix est à mettre en relation avec la peinture des Sarrasins dans l'Estoire. Ceux-ci sont présentés de façon systématique comme polythéistes et idolâtres - erreur grossière que l'on trouve également dans la chanson de geste, l'hagiographie et la littérature polémique. ${ }^{20}$ Comme dans la chanson de geste, les dieux énumérés dans l'Estoire sont Mahomet, Apolin et Tervagant aussi bien que des dieux païens ou des planètes: Jupiter, Mars, le soleil. Ces dieux sont représentés en images dans les sanctuaires des Sarrasins. Il semble que les termes «image » et «deu » (ce dernier étant d'habitude au pluriel) qui désignent les représentations des dieux sarrasins doivent être interprétés comme référant à des sculptures en trois dimensions - forme que les chrétiens associaient avec des idoles païennes. ${ }^{21}$ Pendant tout le Moyen Âge la société chrétienne était préoccupée avec l'idolâtrie ${ }^{22}$; nous reviendrons sur cette question. Il est notable que dans l'Estoire il n'y a jamais de description très développée des statues sarrasines dans les temples et que le jeu des chrétiens va être de prouver leur inefficacité. Dans tous les épisodes où des images apparaissent elles finissent par être brisées, brûlées, détruites.

Prenons comme exemple l'épisode de l'épée brisée. Près de la forêt de Brocéliande (qui est située en Angleterre dans ce texte) Joseph rencontre un Sarrasin qui lui dit «ja n'avons nos que qatre dex: Mahomet et Jupiter et Tervagan et Apollin» (II, 495). Lorsque Joseph lui dit qu'il est médecin - en fait il va jouer un rôle thaumaturge dans cet épisode - le Sarrasin l'emmène dans son château pour guérir son frère. Mais dès leur arrivée, le Sarrasin est attaqué par un lion et meurt. Joseph est alors blessé à la cuisse par le sénéchal du château dont l'épée se brise, laissant une moitié dans la plaie, et est menacé d'emprisonnement. Mais en insistant sur son rôle de médecin, il reste libre. Il fait porter le mort devant les images des dieux sarrasins afin de démontrer leur inefficacité à le ressusciter. Les Sarrasins s'agenouillent devant la statue de Mahomet; après un moment Joseph leur crie que l'image ne peut rien faire, puis il prie Dieu de leur montrer comment ils sont « deceü d'adorer cez maufez.» Suit un coup de tonnerre terrible; la foudre descend sur les images, les brûle et les abat. À la suite d'une autre prière de Joseph, le mort est ressuscité ; tous les habitants du château convertissent et sont baptisés. L'épisode se termine par la guérison du frère du ressuscité par Joseph, qui fait le signe de la croix sur sa plaie, et par l'évocation et la «préparation » de l'épée brisée. Joseph retire de sa cuisse la moitié de l'épée qui y est restée et demande qu'on lui apporte l'autre partie. L'épée sera gardée dans le château; Joseph ne sera guéri qu'ultérieurement. 
19 Dans cet épisode le prosateur insiste sur le polythéisme et l'idolâtrie des Sarrasins. Les étapes de conversion citées pour Évalach sont compressées pour mettre en évidence cet aspect: la conversion de tous les habitants du château est obtenue très rapidement, tout de suite après la démonstration de l'inefficacité des dieux sarrasins et de leurs idoles par rapport au Dieu chrétien. Cette incapacité devient évidente par le bris des idoles.

20 Ce motif, qui parait souvent dans la chanson de geste, est repris dans presque tous les épisodes de conversion de l'Estoire, notamment dans l'histoire de la Croix Noire, qui se passe, comme nous l'avons vu, à Camaalot. La description de cette ville insiste sur son importance comme site sarrasin : c'est «la plus riche cité que sarrazins eüssent en la Grant Bretaigne et estoit de si grant autorité qe li roi paien i estoient coroné et $\mathrm{i}$ estoit la mahomerie plus haute et plus grant qu'a nule autre cité " (II, 479). Comme beaucoup de Sarrasins dans la chanson de geste, le seigneur de cette ville est exceptionnellement cruel et déloyal : «A celui tens que li crestien i vindrent en estoit sires et rois uns hom qui estoit li plus fels et li plus delleaus del monde » (II, 479). ${ }^{23}$

21 Cet épisode présente une variation importante par rapport aux autres : il comporte une conversion feinte; le triomphe du christianisme se fait donc en deux temps. Dans la première partie le prêche de Josephé est aussitôt suivie de la conversion et du baptême d'un grand nombre des habitants. Voyant son peuple adopter la foi chrétienne, Agrestes fait semblant de se convertir afin de mieux combattre cette nouvelle loi. Mais après le départ de Josephé, il convoque ses hommes dans une salle où il a disposé d'une part ses « dieux » et d'autre part «la croix des chrétiens » et leur demande de faire un choix; ceux qui ne veulent pas adorer les dieux sont tués. Ainsi Agrestes fait-il retourner son peuple à la foi sarrasine. Puis il fait martyriser les douze compagnons que Josephé avait laissés à Camaalot et qui refusent d'adorer les dieux sarrasins; la croix où ils sont morts restera noire de leur sang. Quant au roi, il est puni de son outrage, car au moment où il veut faire brûler une autre croix, il devient fou et finit par se jeter dans un four où il meurt. Après sa mort, les habitants de Camaalot rappellent Josephé, qui fait enterrer les martyrs, détruire tous les «temples as paiens» et brûler les idoles; finalement il fait fonder une église en l'honneur de saint Étienne le martyr, édifice qui devient un lieu important du Lancelot et de la Mort Artu. ${ }^{24}$

Dans cette histoire la croix est clairement mise en opposition aux statues des dieux sarrasins, ce qui met en évidence le rôle joué par les signes. L'idolâtrie est condamnée par le bris des idoles et la destruction du temple sarrasin. Cette démolition est suivie de la construction d'une église chrétienne, marquant le triomphe du christianisme. Ce motif se retrouve dans d'autres épisodes. Il représente une étape importante dans la conquête de l'espace et la colonisation par les chrétiens : la ville sarrasine est ainsi purifiée et transformée en lieu chrétien. ${ }^{25}$

L'insistance sur la destruction des idoles et des temples sarrasins se retrouve dans le très long passage consacré à la conversion d'Évalach. Il est notable que les dieux sarrasins énumérés ici sont plutôt des dieux païens et des planètes: Apolin, Mars, le soleil. La séquence consacrée au bris des idoles dans le temple à Sarras démontre aussi l'inefficacité des dieux - dans cette scène ils sont incapables de répondre aux questions posées par Évalach. Mais ils sont aussi associés au diable : un diable dans la statue de Mars explique à Évalach qu'Apolin ne peut pas répondre à ses questions à cause de la présence d'un chrétien qui l'empêche de parler. Le diable commence alors à crier, puis 
il sort de l'image, la renverse et la casse. Enfin il prend un aigle d'or de l'autel du Soleil et frappe l'image d'Apolin, lui cassant le nez et le bras gauche. "Aprés s'en ala par toutes les ymages del temple; si n'i remest onques ymage qui il ne ferist de ichel aigle, tant qu'il li pecheoit aucun des membres » $(\mathrm{I}, 95)$.

Dans cette scène Josephé est présenté comme responsable de la «justice» rendue au diable et donc aux idoles sarrasins. Le bris des images est ici le signe du triomphe futur du christianisme - la conversion d'Évalach n'aura lieu qu'après la guerre décrite dans la séquence narrative suivante. La destruction des temples et la fondation des églises sont donc repoussées à plus tard, après la conversion des habitants des terres d'ÉvalachMordrain et de Seraphe-Nascien. L'épisode se termine par une séquence curieuse où Évalach-Mordrain brûle une sorte de poupée qu'il gardait dans une chambre souterraine secrète. ${ }^{26}$ Bien que le péché du roi soit d'avoir aimé (et couché "carnelement» avec) cette image plutôt que de l'avoir adorée comme dieu, la destruction de cette statue est assimilée à celle des idoles, car elle clôt l'épisode de la conversion d'Évalach.

$\mathrm{Au}$ contraire des statues dans tous les temples, cette image est l'objet d'une description de la part du narrateur: «si estoit une ymage de fust a mervelle de grant biauté en guisse d'une feme et si etoit vestue de si riches reubes com li rois les pooit trover plus riches et plus precieuses » (I, 172-3). Cette statue rappelle les statues de la Vierge dans les églises chrétiennes de cette époque : non seulement étaient-elles polychromes mais elles étaient décorées de bijoux et portaient des vêtements luxueux. ${ }^{27}$ La poupée d'Évalach semble donc être une transposition des images chrétiennes contemporaines que l'on pouvait voir dans toutes les églises à l'époque. Pourtant cette sorte d'image est absente de l'Estoire: il n'y a aucune mention de statues dans les églises chrétiennes qui sont érigées pour remplacer les temples sarrasins. La seule image chrétienne dans l'Estoire est la Véronique - image du Christ en deux dimensions - qui guérit Vespasien, le fils d'un empereur romain, de la lèpre. Rappelons que c'est Vespasien qui libère Joseph d'Arimathie, emprisonné après la disparition du corps du Christ. Pendant une grande partie du Moyen Âge les chrétiens distinguaient entre des images en deux et trois dimensions; ces dernières leur semblaient plus dangereuses et donc plus près de l'idolâtrie. ${ }^{28} \mathrm{Il}$ est donc significatif que la seule représentation chrétienne dans l'Estoire est en deux dimensions.

Il faudrait comparer toutes ces images à la statue d'Ypocras évoquée dans un récit rétrospectif où le médecin grec guérit le neveu d'un empereur romain que l'on croit mort. Par reconnaissance, l'empereur fait faire "une grant figure d'or et haute en figure d'ome semblable au plus que l'en pot en la forme d'Ypocras, et aprés refist faire une autre a la maniere de son neveu, puis les fist metre desus la mestre tor de Rome, el plus haut liu si que nus n'entrast en la vile qui nel peüst veoir apertement » (II, 350). Les Romains révèrent la statue d'Ypocras comme un dieu, mais le pouvoir du médecin de ressusciter un mort est mis en doute par une femme qui finit par le confondre. L'empereur fait alors abattre les statues. Cette histoire exemplaire condamne donc les prétentions du médecin à un pouvoir miraculeux qui rivalise avec celui du Christ en même temps que l'idolâtrie. La statue d'Ypocras et la poupée d'Évalach sont les seules images décrites en détail dans l'Estoire. Nous avons vu la ressemblance de cette poupée avec des images de la Vierge. La statue d'Ypocras, qui est en or, ressemble à des sculptures païennes que l'on pouvait voir à l'époque mais aussi à certaines images de saints contemporains. ${ }^{29}$ 

Guillaume, par exemple, les Sarrasins qui assiègent la ville de Narbonne y transportent une énorme "image » de Mahomet. ${ }^{30}$ On trouve une description assez développée de cette statue qui souligne sa richesse - elle est en or - et sur les couleurs et les lumières qui l'entourent. L'insistance sur la richesse des idoles, qui est un thème fréquent, est une façon de les condamner. ${ }^{31}$ Sous le regard d'abord effrayé puis amusé des chrétiens, qui montent sur les murs crénelés de la ville, les Sarrasins lui font des offrandes. Puis un Sarrasin entre dans la statue et la fait parler et bouger ; les Sarrasins croient alors à la victoire. À ce moment-là les chrétiens commencent à jeter des pierres et d'autres projectiles, brisant l'image en la renversant et tuant le Sarrasin qui se cache à l'intérieur. Quand le chef, Thiebaut, voit la statue par terre, il l'insulte : «He, Mahomet, cent dehais aiez tu,/Car tes vertus ne valent un festu !» (vv 1575-76) Cette destruction, qui sert à ridiculiser les Sarrasins et leurs croyances, est le prélude à la victoire des chrétiens contre leurs assiégeants.

Dans la chanson de geste le bris des idoles, qui est une scène fréquente, marque souvent la victoire militaire des chrétiens sur les païens et donc symbolise le triomphe de la religion chrétienne sur la foi sarrasine. Dans la deuxième partie de la Chanson de Roland, par exemple, après avoir conquis Baligant, Charlemagne prend Saragosse où les chrétiens brisent les idoles et font baptiser les païens. Ceux qui refusent la foi chrétienne sont tués. La seule exception est la reine Bramimonde, qui est emmenée captive en France, car Charles veut qu'elle se convertisse "par amur. ${ }^{32}$ L'emploi de la violence coexiste avec la persuasion, qui est cependant réservée à une seule personne. La conversion est liée à l'échec guerrier : le perdant a le choix de la conversion ou la mort.

Dans les Enfances Guillaume les Sarrasins qui assiègent Narbonne sont défaits au cours d'un massacre général ; ceux qui le peuvent s'enfuient sans tentative de conversion. La conversion est liée plutôt à la conquête future d'Orange - et d'Orable, la reine de cette ville, conversion qui se prévoit dans ce texte. Orable, déjà amoureuse de Guillaume sans l'avoir jamais vu mais ayant entendu parlé de ses prouesses, dit à son frère qui veut la marier à Thiebaut : «A poc Guillaume ne m'ait ja convertie/Et destorné de la paienime,/ Si croirai Deu, le fil Sainte Marie » (vv. 1745-47).

30 Le massacre des Sarrasins, thème fréquent dans la chanson de geste, est justifié par leur caractérisation comme infidèles, cruels et déloyaux. Dans l'Estoire, où la conversion est obtenue par la persuasion plutôt que par la violence, ce genre d'hécatombe existe aussi. Lorsque le roi de Northumberland assiège son vassal, le duc Ganor, avec l'intention de le faire renoncer à la foi chrétienne, les chrétiens massacrent les Sarrasins. Après la mort du roi, qui préfère mourir comme sarrasin que vivre comme chrétien, ses hommes essaient de s'enfuir : «tornerent maintenant en fuie li plusor et s'en fuirent parmi le Hombre, tuit a cheval. Si en i ot mout de noiez et periz, einz qu'il fussent outre ; cil qui passerent furent gari, mes sachiez qu'il en i ot peu, car si pres les tenoient cil de Galefort qu'il les abatoient en l'eve, si come il les pooient aconsivre » (II, 452).

31 La peinture des Sarrasins dans l'Estoire ressemble donc beaucoup à celle que l'on trouve dans la chanson de geste. Le travail de réécriture de l'auteur anonyme de l'Estoire comporte une multiplication des épisodes où Joseph et Josephé convertissent des Sarrasins et luttent contre leur religion idolâtre. Comme dans la chanson de geste, ils sont condamnés surtout pour leur polythéisme idolâtre - erreur grossière fondée dans 
l'idéologie. Dans son étude des idoles dans l'iconographie chrétienne à l'époque "gothique » (du milieu du XII ${ }^{e}$ siècle à la fin du XIV siècle), M. Camille démontre que la représentation des idoles reflète une nouvelle préoccupation concernant les images. À cette époque les chrétiens d'Occident, qui utilisaient de plus en plus d'images dans les cathédrales et dans les livres pour la dévotion personnelle, cherchaient à justifier cette pratique par des passages dans la Bible. Puisque d'autres passages bibliques condamnaient la représentation en images et l'idolâtrie, il fallait trouver le moyen de distinguer entre les bonnes et les mauvaises images. Ils ont contourné le problème en appelant leurs propres représentations des «figures» des êtres divins. ${ }^{33} \mathrm{La}$ stigmatisation des idoles qu'ils attribuaient à la religion musulmane qu'ils affirmaient être l'objet d'une vénération en elles-mêmes, servait donc à transférer sur l'autre un malaise devant le problème de l'idolâtrie. La condamnation des idoles sarrasines peut être vu en fait come une sorte de mécanisme d'auto-défense, car on pouvait adresser aux chrétiens la même critique qu'ils faisaient des Sarrasins : le culte des saints, en particulier, a des traits qui ressemblent beaucoup au polythéisme idolâtre.

Cette préoccupation avec l'idolâtrie est présente dans l'iconographie du XIII ${ }^{e}$ siècle où l'un des thèmes les plus importants est le bris des idoles. On peut citer en particulier un épisode de la vie de l'enfance du Christ, la chute des idoles lors de la fuite en Égypte, épisode raconté par le pseudo-Mathieu. Dès que l'enfant Jésus est arrivé dans un temple dans la ville de Sotinen, les idoles sont tombées par terre et se sont brisées, accomplissant une prophétie d'Isaïe. Cette scène, qui est fréquemment représentée, démontre le triomphe du Christ et de la nouvelle loi sur l'idolâtrie. ${ }^{34}$

Il est notable que, malgré l'importance des idoles dans l'Estoire, ce thème semble absent du programme iconographique des manuscrits. Les manuscrits de la Bibliothèque Nationale datés du XIII ${ }^{\mathrm{e}}$ siècle ne contiennent aucune représentation d'idoles. ${ }^{35}$ L'accent est mis plutôt sur les scènes qui présentent des aspects de la doctrine chrétienne comme la Crucifixion ou sur des scènes liées à la conversion des Sarrasins ou les épreuves des nouveaux chrétiens. C'est comme si l'idolâtrie faisait tellement peur que l'on ne pouvait pas représenter les idoles. M. Camille constate les effets de cette peur qui peut amener l'artiste à laisser une place vide où une idole doit être placée, ou bien un propriétaire/lecteur de manuscrit à effacer l'idole ou encore la destruction de

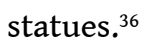

\section{NOTES}

1.Lancelot, éd., A. Micha, 9 tomes, Genève, Paris, Droz, 1978-83, II, 321. E. Baumgartner attire l'attention sur la présence des Sarrasins dans le Lancelot, dans « Joseph d'Arimathie dans le Lancelot en prose ", Actes du colloque des 14 et 15 janvier 1984. Lancelot, éd. D. Buschinger, Goppingen, Kummerle Verlag, 1984, pp. 7-15. Voir aussi son article "Géants et chevaliers ", The Spirit of the Court, éd. G. S. Burgess et R. A. Taylor, Cambridge, D.S. Brewer, 1985, pp. 9-22.

2.Pour la liste des occurrences, voir Baumgartner, « Joseph d'Arimathie », note 1. 
3.L'Estoire del Saint Graal, éd. J-P. Ponceau, 2 tomes, Paris, Champion, 1997, II, 417. Au moment où les chrétiens arrivent à Camaalot, on trouve cette description : « A celui tens n'avoit il encore en Camaalot ne en tote la contree se mescreanz non » (II, 479). 4.La plupart des chercheurs pensent que l'Estoire a été rédigée après le Lancelot et après la Queste del saint Graal. Voir Michelle Szkilnik, qui résume les positions dans le débat jusqu'alors dans L'Archipel du Graal. Étude de l'Estoire del Saint Graal, Genève, Droz, 1991, p. 1, n. 1. J-P. Ponceau pense au contraire que l'Estoire a été écrite avant la Queste, L'Estoire del Saint Graal, Thèse de doctorat, 1989, 3 tomes, III, 366-406.

5.Sur la réécriture voir E. Jane Burns, Arthurian Fictions. Rereading the Vulgate Cycle, Columbus, Ohio State Univ. Press, 1985, ch. 1, et E. Baumgartner, « L'écriture romanesque et son modèle scripturaire : écriture et réécriture du Graal », L'Imitation. Colloque de l'École du Louvre, Paris, La Documentation Française, 1984, pp. 129-143. Dans son étude de l'Estoire, M. Szkilnik suggère plusieurs métaphores pour décrire la composition - la bête diverse, l'arbre à plusieurs branches et l'aimant, avant d'adopter celle de l'archipel, L'Archipel du Graal. pp. 6-8. Elle signale que le roman comprend presque tous les genres littéraires, pp. 111-115.

6.Le passage se trouve dans Micha, II, 325-339.

7.L'histoire se trouve dans Ponceau, II, 494-501.

8.Sur les changements que l'auteur de l'Estoire opère et les autres sources, voir F. Lot, Étude sur le Lancelot en prose. Augmenté d'un septième appendice dû à M. Lot-Borodine, Paris, Champion, 1984, pp. 204-214, et Szkilnik, op. cit., pp. 71-73.

9.Baumgartner, «Joseph d'Arimathie », p. 12.

10.Lorsque Joseph s'inquiète de comment il va convertir Évalach, Dieu lui répond qu'il le fera «pareil a mes apostles par les miracles et par les virtus ke je ferai par tes mains » (I, 43). Sur le rôle de Joseph dans l'Estoire voir Szkilnik, op. cit., p. 71 et C. Chase, «La conversion des païennes dans l'Estoire del Saint Graal, » Arthurian Romance and Gender/Masculin/Féminin dans le roman arthurien médiéval /Geschlechterrollen im mittelalterlichen Artusroman, éd. F. Wolfzettel, Amsterdam et Atlanta, Rodopi, 1995, p. 255.

11.F. Lot signale l'importance de cette « trouvaille », Étude sur le Lancelot, p. 205.

12.Le cycle du Lancelot-Graal a été écrit pendant le premier tiers du XIII ${ }^{\mathrm{e}}$ siècle. On peut situer la date de l'Estoire aux alentours de 1220. Voir A. Stones, « The Earliest Illustrated Prose Lancelot Manuscript ", Reading Medieval Studies, III, 1997, pp. 3-44. Les dates de composition des chansons de geste sont assez incertaines, mais il semble que les Enfances Guillaume, dans son état actuel, date du début du XIII ${ }^{\mathrm{e}}$ siècle. Voir B. Guidot, Recherches sur la chanson de geste au XIII ${ }^{e}$ siècle, 2 tomes, Aix-en-Provence, Pub. Univ. de Provence, 1986, I, Xv-xxxii. Nous tenons à remercier Caroline Cazanave pour son aide précieuse en ce qui concerne des sources bibliographiques pour la chanson de geste. 13.P. Bancourt, Les Musulmans dans les chansons de geste du cycle du roi, Aix-en-Provence, Pub. de l'Université de Provence, 1982, pp. 1-2 et 341-570. Voir aussi Guidot, op. cit., pp. 72-73 et 84-87.

14.Szkilnik, op. cit., pp. 30-31.

15.Op. cit., pp. 63-65. Cette critique met l'accent sur l'Incarnation et sur l'Immaculée Conception. Mais l'Estoire insiste sur la virginité de Marie avant et après la naissance du Christ, dogme qui a été mis en place au XIII ${ }^{\mathrm{e}}$ siècle.

16.Tout au long de l'Estoire le numéro trois va jouer un rôle très important. Sur le prologue de l'Estoire voir A. Leupin, Le Graal et la littérature. Étude sur la Vulgate 
arthurienne en prose, Lausanne, L'Age d'Homme, 1982, pp. 21-35, et R. Pickens, "Autobiography and History in the Vulgate Estoire and in the Prose Merlin », The Lancelot-Grail Cycle. Text and Transformations, Austin, Univ. of Texas Press, 1994, pp. 98-116.

17.Szkilnik, op. cit., pp. 65-66.

18.Dans la chanson de geste il y a parfois des débats; on y trouve la même préoccupation avec la Trinité et la naissance virginale du Christ et des méthodes d'argumentation similaires. Voir Bancourt, op. cit., pp. 474-492.

19.Rappelons que Josephé couvre la croix avec un tissu blanc qu'Évalach doit enlever seulement au moment où il a peur de la mort; qu'un chevalier blanc (le Christ) apparaît à ce moment critique et délivre le roi ; que la croix de cet écu guérit un chevalier dont la main a été coupée et que la croix est transférée sur le bras de celui-ci ; qu'Évalach emporte l'écu en Grande Bretagne ; qu'au moment de mourir Josephé y trace une nouvelle croix rouge avec son propre sang; et finalement que l'écu va rester dans l'abbaye où Mordrain attend l'arrivée de Galaad, à qui il est destiné.

20.Bancourt, op. cit., pp. 357, 391-2.

21.M. Camille, The Gothic Idol, Cambridge, Cambridge Univ. Press, 1989, pp. 41-43. Sur le terme « image » voir aussi Bancourt, op. cit., p. 387.

22.Camille, op. cit., p. 36 et passim.

23.Bancourt, op. cit., pp. 113-115 ; Guidot, op. cit., p. 64. Dans l'Estoire les souverains sarrasins qui n'adoptent pas la foi chrétienne sont caractérisés de cette façon ; même ceux qui convertissent peuvent agir de façon cruelle avant leur conversion.

24.La destruction du temple sarrasin et l'édification de cette église ne sont pas rapportées dans l'histoire de la Croix Noire du Lancelot. L'auteur de l'Estoire rajoute donc cet élément important. Il insiste aussi sur l'importance de Camaalot comme site sarrasin ; la description citée ci-dessus ne figure pas dans la version de cette histoire du Lancelot.

25.Voir Szkilnik, op. cit., pp. 30-33.

26.Sur cette " poupée » voir Colette-Anne Van Coolput, « La Poupée d'Evalac ou la conversion tardive du Roi Mordrain », Continuations. Essays on Medieval French Literature and Language in honor of John L. Grigsby, éd. N. Lacy et G. Torrini-Roblin, Birmingham, Alabama, Summa, 1989, pp. 163-172.

27.Camille, op. cit., pp. 227-229.

28.Ibid., pp. 41-43.

29.Bancourt décrit deux modèles pour les idoles dans la chanson de geste : la statue d'Hercule à Cadix, détruite au XII ${ }^{e}$ siècle, op. cit., p. 394, et la statue de sainte Foy de Conques, op. cit., pp. 402-403. Voir aussi Camille, op. cit., p. 41.

30.Le passage se trouve dans P. Henry, éd ; Les Enfances Guillaume, Paris, SATF, 1935, vv. 1509-1585. L'épisode figure dans l'anthologie de D. Boutet, Le Cycle de Guillaume d'Orznge, Paris, Poche (Collection Lettres Gothiques), 1996, pp. 46-51.

31.Dans Psaumes 113, 4, les idoles sont condamnées parce qu'elles sont en argent et en or et parce qu'elles sont fabriquées par les mains d'hommes. Voir Camille, op. cit., p. 9. 32.Gérard Moignet, éd. et trad., La Chanson de Roland, Paris, Bordas, 1972, vv. 3660-3674. 33.Camille, op. cit., pp. 203 sq.

34.Ibid., pp. 1-3. L'historien de l'art montre que la façon de traiter la représentation de cet épisode change au XIII ${ }^{\mathrm{e}}$ siècle pour insister sur le côté négatif des idoles (p. 4). 
35.Nous préparons une étude de l'Estoire qui tiendra compte de la relation entre le texte et les enluminures. La seule image que nous avons trouvée representée dans les manuscrits du XIII siècle est la Véronique (BN 749 et 9123). Sur l'iconographie de l'Estoire voir A. Stones, art. cit., et M. Meuwese, « Twelve Bleeding Tombs and Seven Flaming Hands : Text and Image in the Amsterdam Estoire », The Arthurian Yearbook, II, 1992, pp. 135-158.

36.Camille, op. cit., pp. 18-23.

\section{AUTEUR}

CAROL CHASE

Knox College 